\title{
29. STELLAR SPECTRA (SPECTRES STELLAIRES)
}

\author{
President: M.S. Bessell \\ Vice-President: B. Barbuy \\ Organizing Committee: A. Boesgaard, P. Cottrell, M. Gerbaldi, D. Gray \\ G. Mathys, R. Peterson, N. Rao, K. Sadakane, G. Zhao
}

Introduction. Over the past 3 years the availability of abstracts, journal's table-of-content, bibliographic searches and even preprints on the Internet means that summaries such as this will be unnecessary. The AAS WWW bibliographical/abstract services (http://cfa-www.harvard.edu/aas/) accesses several useful sites: http://cfa-www.harvard.edu/aas_search.shtml for bibliographic searches of ApJ, ApJL, ApJS, AJ and PASP from 1988 to the present. The ADS abstract bibliographic service covering in addition A\&A, A\&AS, MNRAS, PASJ, RMexAyA, PASAus, IrishAJ, BASInd, BalAst and other journals is http://adsabs.harvard.edu/abstract_service.html; http://adsabs.harvard.edu/toc_service.html is for table-of-content queries that are useful for browsing the same journals. Most of these services are also available through the CDS (http://cdsweb.u-strasbg.fr/CDS.html) in several different languages. From this site can also be obtained published tables from astronomical journals as well as other data that have been deposited by individuals. The SIMBAD database (http://cdsweb.u-strasbg.fr/Simbad.html) maintains its importance as a stellar database in particular. It is necessary to have a user account to access SIMBAD. The ApJ contents is now online at http://www.journals.uchicago.edu/ApJ/journal/contents/ApJ . Notable by its absence from these data bases is Nature from whose home page (http://www.nature.com) the TOC of the current year's issues only can be browsed after registering. Preprints can be obtained from http://xxx.lanl.gov/archive/astro-ph covering the years 1992 to the present. The various search engines also often turn up papers given at conferences or in other publications than the mainstream astronomical journals. Transition probabilities and term levels are an important area long neglected for the provision of digital data (with the honourable exception of CDROM 18 from R.L.Kurucz). The spectroscopic databases of the NIST (http://aeldata.phys.nist.gov/nist_atomic_spectra.html) now contain extensive data for some elements, in particular the transitional probabilities for the iron group elements. One assumes that the list of elements with gf values will be progressively increased. It is obvious from a perusal of these web sources as well as noting the rapid filling of library shelves, that the number of papers being published in astronomy and astrophysics continues its inexorable increase. This makes it even more difficult to do justice to all the work done over the past 3 years.

As usual, new instruments make their mark and amongst the highlights this year are significant spectroscopic observations made with the HST and with the Keck high resolution spectrograph. The next report will surely include more of such observations and also highlight the $2 \mathrm{dF}$ fibre spectrograph at the AAT which will enable a large number of stars to be measured simultaneously.

This report of highlights associated with spectroscopy has been made with the assistance of colleagues who kindly sent me lists of papers that had excited them over the past three years. I thank R. Bell, F. Castelli, G. Mathys, B. Pagel, HM Schmid, R. Wehrse, B. Wolf for their suggestions and R.E.M Griffin for her report on the Working Group on Spectroscopic Data Archives.

Model Atmospheres. Model atmospheres grids continue to expand their parameter space. A review of late-type stellar photospheres was given by Gustafsson and Jorgensen (1994, A\&ARev 6, 19). Following the cool opacity sampled giant models (Plez et al. 1992, A\&A 256, 551), Brett published opacity sampled dwarf and subdwarf models (Brett 1995 AA 295, 736; A\&AS 109, 263). These produce excellent spectra and colors for stars between $3000 \mathrm{~K}$ and $4250 \mathrm{~K}$ (Bessell 1994, in "The Bottom of the Main Sequence - And Beyond", ed. C.G. Tinney, Springer, p123) connecting with the Kurucz (1993, CDROM 18) model colors for temperatures above 4250K. Allard and Hauschildt (1994, in "The Bottom...", loc cit., p32; 1995, ApJ $445,433)$ have also produced grids of excellent line blanketed cool dwarf atmospheres. The model regime between planetary atmospheres and brown dwarfs involving many polyatomic molecules ignored in studies of hotter stars has been studied by Tsuji et al (1995, A\&A 305, L1; 1996, A\&A 308, 29). They show that dust formation is important in the coolest $\mathrm{M}$ dwarfs and it strongly affects the temperature structure 
and spectra; however, in the $1000 \mathrm{~K}$ model which fits an observed brown dwarf's spectrum, the effects of dust are absent suggesting that dust may have formed into clouds and not remain dispersed within a homogeneous atmosphere. The effects of two important molecules on cool model atmospheres have been investigated; $\mathrm{TiO}$ by Jorgensen $(1994, \mathrm{~A} \& \mathrm{~A} 284,179)$ and $\mathrm{H}_{2} \mathrm{O}$ by Jones et al. (1995, MNRAS 277, 767). Bessell et al. (1996, A\&A 307, 481) continue their investigation of the phase and cycle dependence of the photospheric structure of self-consistent models of mira variables.

The models of Kurucz 1993 (CDROM-18) continue to be the most widely used, however these are in the process of being recomputed using improved opacities and modified physical assumptions; Castelli, Gratton, Kurucz (1996, A\&A in press) discuss the treatment of convection in ATLAS9. In an important move, Castelli (1996, private communication) has recomputed the ATLAS9 models for temperatures below $8500 \mathrm{~K}$ with "approximate overshooting" switched off. These new models produce hydrogen lines and colors that are in excellent agreement. (See comments below concerning papers by Axer et al.)

The UBVRI colors of the grid of Castelli ATLAS9 models, the Plez et al. giant models and the M dwarf models of Brett are discussed in Bessell, Castelli \& Plez (1996 preprint). Bell \& Tripicco (1995, AJ $110,3035)$ calculate indices for models of M67 stars which fit quite well.

Gustafsson, Plez, Nissen and Nordlund have started computing a new grid of opacity sampled models for temperatures between $8500 \mathrm{~K}$ and $2500 \mathrm{~K}$. Becker and Butler $(1995$, A\&A 301, 187) have extended their non-LTE analysis to Fe IV. The non-LTE formation of LiI in cool stars was examined by Carlsson et al $(1994$, A\&A 288, 860) who provide correction tables and Kiselman (1994, A\&A 286, 169) studied the non-LTE formation of BI lines in solar-type stars finding a correction of +0.56 dex for the metal-poor star HD140283 and +0.4 dex for Procyon. The sun was unaffected. Other papers dealing with $\mathrm{Li}, \mathrm{Be}$ and $B$ in stellar atmospheres will be discussed later.

In another cautionary tale, Gonzalez and Lambert (1996, AJ 111, 424) compare the Fe abundances in a F5Ib supergiant with those of FG dwarfs in the same cluster and find a difference of about -0.4 dex which they ascribe to non-LTE effects in the supergiant.

Hauschildt et al. (1994, ApJ 422, 831; 1995, ApJ 447, 829) have used their non-LTE expanding model atmospheres to investigate the spectra of Nova outbursts, and in a significant breakthrough, Baschek, Grüber, von Waldenfels, Wehrse (1996, A\&A in press) have found an analytical solution of the comoving frame radiative transfer equation for a differentially moving slab. Compared to conventional algorithms, it allows speed-up factors of $>10^{5}$ in the calculation of wavelength integrated quantities such as total flux and energy balance.

Temperatures, Bolometric Corrections. There have been several advancements in the empirical temperature scales of stars cooler than $9000 \mathrm{~K}$ and controversy surrounds the application of theoretical model atmospheres to temperature derivations and abundances.

Blackwell and Lynas-Gray (1994, AA 282, 899) present an updated list of stellar effective temperatures using the IRFM and new Kurucz models. Megessier (1994, A\&A 289, 202; 1995, A\&A 296, 771) discusses the influence of models on the IRFM temperatures and the accuracy of absolute visible fluxes.

In a series of papers, Axer et al. (1994, A\&A 291, 895; 1994, A\&A 285, 585; 1995, A\&A 300, 751; 1995, A\&A 301, 492) discuss problems with model atmospheres of FG subdwarfs causing significant systematic errors in analyses. See above for possible solution from Castelli; however, Kurucz (1995, ApJ 452, 102) suggests that apart from problems with the normal temperature stratifications in models, inhomogeneities in the atmospheres of Pop II stars hotter than $5500 \mathrm{~K}$ can underestimate the derived abundance of LiI by a factor of 10 .

Most recently, Van'T Veer-Menneret and Megessier (1996, A\&A 309, 879) discuss effective temperatures of $\mathrm{A}$ and $\mathrm{F}$ stars from Balmer line profiles. Gratton et al $(1996, \mathrm{~A} \& \mathrm{~A} 314,191)$ present a new $\mathrm{T}_{\text {eff }}$ scale for metal-poor stars. Alonso et al (1996, A\&A 313, 873) present an empirical temperatures scale for F0V - K5V stars. Quirrenbach et al. (1996, A\&A 512, 160) derive the effective temperature for Arcturus of $4303 \pm 47 \mathrm{~K}$ from radii measurements. And, radii and effective temperatures for $\mathrm{K}$ and $\mathrm{M}$ giants have been derived by Dyck et al. (1996, AJ 111, 1705).

Balona (1994, MNRAS 268, 119) gives effective temperatures, bolometric corrections and masses for $\mathrm{O}-\mathrm{F}$ stars; while bolometric fluxes have also been derived for 118 dwarfs and subdwarf between $4000 \mathrm{~K}$ and $7000 \mathrm{~K}$ by Alonso et al $(1995, \mathrm{~A} \& \mathrm{~A} 297,197)$.

Spectrophotometry. Very precise spectrophotometric standards have been established by Hamuy et al. (1994, PASP 106, 566). The telluric bands have been removed from these fluxes by Bessell (1996, PASP in press) making them more useful for flux calibration in the red. Spectrophotometric standards 
from the far-UV to the near-IR have been published by Bohlin (1996, AJ 111, 1743). Alekseeva et al. $(1997$, BaltA 6, 1) have published the Pulkovo Spectrophotometric Catalog of $252 \mathrm{HR}$ stars from the southern and northern hemisphere. The wavelength coverage is from 320 to $1080 \mathrm{~nm}$ for most stars.

Techniques. It has always been a holy grail of spectroscopy to be able to derive accurate stellar parameters from low to medium resolution spectra. Such attempts have usually been thwarted by the non-uniqueness of the solutions and limitations in the synthetic spectra. However, given knowledge of the effective temperatures of the stars and by using some comparison stars with known abundances, excellent results can be obtained. Recent papers push the limits of this technique.

Jones et al. (1996, MNRAS 278, 146) discuss using moderate resolution fibre-fed spectroscopic data for abundance analyses and Cuisinier et al. (1994, A\&A 285, 943) discuss fitting low resolution spectra with synthetic spectra to derive abundances. Valenti and Piskunov (1996, A\&AS 118, 595) describe a software package for best fitting observed and synthetic spectra and solving for stellar parameters of $\mathrm{T}_{\text {eff }}, \log \mathrm{g}$, abundances, or atomic data. In a similar vein, von Hippel et al. (1994, MNRAS 269, 97) show their initial results with artificial neural networks to automate the classification of stellar spectra.

Techniques for measuring precise stellar temperatures have been developed and refined by Gray (1994 PASP 106, 1248; 1995 PASP 107, 120). Basically one uses the ratios of the central depths of lines measured on high signal-to-noise exposures of the spectra. This technique has been used on the sun (see below) and on several cool dwarfs, and temperature variations of several degrees are seen for some of them. These stars also typically show variations in magnitude, color, chromospheric emission, and granulation (from the spectral line bisectors). The same pattern of variation is seen in each variable, but there are time lags, and the time lags appear to depend on spectral type (Gray 1994 PASP 106, 145). Some stars show no such variations. These results appear in a series of papers, the most recent of which are Gray et al. (1996 ApJ 465, 945; 1996 ApJ 456, 365).

Precise temperature variations measured from the equivalent width variations in $\mathrm{H} \alpha$ are used by Bedding et al.(1996, MNRAS 280,1155) in a new technique to detect stellar oscillations.

Papers of spectroscopic observations with the Hopkins Ultraviolet Telescope in the wavelength region 900-1800A appeared in the special issue of ApJL 454, L1-72, 1995.

Clegg, Storey, Walsh \& Neale (1996, ESO-Preprint No.1186, MNRAS) have measured the ${ }^{12} \mathrm{C} /{ }^{13} \mathrm{C}$ abundance ratio in planetary nebulae with HST using the ${ }^{13} \mathrm{C}[\mathrm{III}] 190.96 \mathrm{~nm}$ hyperfine transition.

Spectropolarimetric structure in the Raman lines has been studied by Schmid \& Schild (1994, A\&A $281,145)$ and Harries \& Howarth (1996, A\&AS 119, 61).

Larson and Irwin (1996, A\&AS 117, 189) have developed an efficient method for dealing with the multitude of weak lines that depress the true continuum. They apply this to the 864 to $878 \mathrm{~nm}$ wavelength region containing many molecular lines in cool stars.

Groot et al. (1996, A\&AS 118, 545) apply the Fourier-Bessel transformation method to derive the rotational velocities of 178 bright dwarfs with spectral types between A2 and G2.

Sky subtraction with fibre-spectrographs is discussed by Lissandrini et al. (1994, PASP 106, 1157). They find that the [OI] line at $557.7 \mathrm{~nm}$ makes the best flat field calibrator.

Valenti et al. (1995 PASP 107, 966) investigated the instrumental profile of their echelle spectrograph and show how it varies across the field.

Spectroscopic Atlases. Useful stellar atlases were been published during the past 3 years, some of them in the UV and IR.

An atlas of OB spectra from 100 to $120 \mathrm{~nm}$ : Walborn and Bohlin (1996, PASP 108, 477). IUE spectra $(116$ to $189 \mathrm{~nm}$ ) of OB stars: Bohlin et al. (1994, A\&AS 105, 305). A high resolution UV spectrum (195 to $200 \mathrm{~nm}$ ) of the Sun: Samain (1995, A\&AS 113, 237). An Atlas of Ultra-Violet P Cygni Profiles: Snow et al. (1994, ApJS 95, 163).

Southern MK standards (580 to $1020 \mathrm{~nm}$ ): Danks and Dennefeld (1994, PASP 106, 382). A moderateresolution spectral atlas of carbon stars: $\mathrm{R}, \mathrm{J}, \mathrm{N}, \mathrm{CH}$ and barium stars: Barnbaum et al. (1996, ApJS 105, 419). An atlas of optical spectra of Galactic WN stars: Hamann et al. (1995, A\&AS 113, 459). A related database of equivalent widths in WR stars: Niedzielski et al. (1994, A\&AS 108, 669). An atlas of high resolution line profiles of symbiotic stars: Iveson et al. (1994, A\&AS 103, 2100).

An infrared (0.9 to 5.3 microns) atlas of Arcturus: Hinkle et al. (1995, PASP 107, 1042). An infrared ( 1 to 2.5 microns) atlas of $M$ dwarfs: Jones et al. (1996, MNRAS 267, 413). A library of $\mathrm{H}$ band spectra of class I, III and V stars: Dallier et al. (1996, A\&AS 116, 239). Finally, a night-sky spectral atlas from the Keck HIRES spectrograph: Osterbrock et al. (1996, PASP 108, 277). 
Doppler imaging. There was less activity in this field than previously but an interesting paper is by Rice \& Wehleu (1994 PASP 106, 817) where they map chemical spots on 84 UMa. Unruh and Collier Cameron (1995, MNRAS 273,1) examine the sensitivity of such imaging against errors in the line profile.

Magnetic, $\mathrm{Cp}$ and related stars. Work on these topics continues to attract significant interest from several groups. Highlights include the preprint by Mathys et al. (1996, A\&AS, in press; ESO preprint No. 1194) which presents the largest series of original stellar magnetic field measurements since Babcock's (1958, ApJS 3, 141) famous catalogue and which allows several new general properties of Ap stars to be evidenced. Another important paper by Abt \& Morrell (1995, ApJS 99, 135) discusses the relation between rotational relocities and spectral reculiarities among A-type stars. Adeleman (1996, MNRAS 280,130 ) continued analyses of Ap stars and superficially normal late-B and early A stars in one of the more recent papers. Smith continued studies of the IUE spectra of Ap stars; (Smith 1994, A\&A 291, 521; 1995, A\&A 297, 237). Amongst other papers are Babel (1994, AA 283, 189) - Ca stratification in Ap stars; Hatzes \& Kuerster (1994, A\&A 285, 454) - rapid radial velocity variations in $\alpha$ Cir; Gonzalez \& Artru (1994, A\&A 285, 454) - O lines in Ap stars; Rice \& Wehlau (1994, A\&A 291, 825) - surface distribution of $\mathrm{Fe}$ and $\mathrm{Cr}$ in $84 \mathrm{UMa}$; Hubrig et al. (1994, A\&A 291, 890) - a search for magnetic fields in late-type stars; Leone et al. (1994, A\&A 293, 457) - a search for hot stellar envelopes in magnetic peculiar stars using the 10830 line of HeI; Mathys G. (1995, A\&A 293, 733; A\&A 293, 746) - spectropolarimetry of magnetic stars; Mathys \& Hubrig $(1995$, A\&A 293, 810) - magnetic fields on HgMn binaries; Wade et al. (1996, A\&A 313, 209) - magnetic model for HD 192678.

Hot stars. One of the most interesting aspects of hot star research concerns the stellar winds and variability. The 16 day IUE MEGA Campaign proved extremely successful and the results were discussed in four papers in ApJL 452 in 1995. Massa et al. p53; St-Louis et al. p57; Prinja et al. p61; and Howarth et al. p65). The ground based campaign also yielded interesting results (Kaufer et al.1996, A\&A 305, 887; Kaufer et al. 1996, A\&A 314, 599).

In the WN5 star HD 50896 (EZ CMa), the observed variations in the emission lines suggest a global wind structure pattern that remains quite stable in the frame of the star, over the duration of the observations. This pattern was marginally compatible with that expected for the ionization cavity around an accreting neutron star companion in a 3.766 day W-R + NS binary. However, it can best be explained by some kind of corotating interaction regions emanating from hot (magnetically?) active regions near the surface of the stellar core. Such a model, although somewhat ad hoc, better accounts for the strong epoch-dependent variations seen in EZ CMa, as well as the fine structures seen in the global pattern.

In the B0.5Ib star HD64760, the extensive coverage revealed a pattern of rapidly evolving discrete optical depth changes which typically migrate from $-200 \mathrm{kms}^{-1}$ to $-1500 \mathrm{kms}^{-1}$ in less than $12 \mathrm{hr}$. These features coexist with more slowly evolving structures lasting several days. The authors claim the data is consistent with an interpretation in terms of a set of rotationally modulated wind variations in HD 64760 . The basic result of very regular, large-scale optical depth variations points to a "clock" whose origin is on the stellar surface, rather than a mechanism that is entirely intrinsic to the stellar wind.

The variations in the SiIV doublet of the $\mathrm{O} 4 \mathrm{I}(\mathrm{n}) \mathrm{f}$ star $\zeta$ Pup was also followed. The data show continuous variability throughout the greater part of the blueshifted absorption. Time series analysis of these data revealed significant power at periods of $19.2 \mathrm{hr}$ and 5.2 days, which were identifed with the mean recurrence time of "discrete absorption components" (DACs) and the photospheric rotation period, respectively. These results indicate that the wind has a global longitudinal asymmetry (approaching a factor 2 in optical depth), possibly associated with large-scale magnetic structures, but suggest that the DACs are not directly associated with specific stellar longitudes in this star. There was no significant power in the lines at the $8.5 \mathrm{hr}$ period identified in photospheric absorption-line variability, nor at the $16.7 \mathrm{hr}$ period reported in X-ray observations.

Puls et al. (1996, A\&A 305, 171) discussed observations and theoretical predictions of O-star mass-loss and wind momentum rates in the Galaxy and the Magellanic Clouds

In another interesting paper, Lamers, Snow and Lindholm (1995, ApJ 455, 269) measured the terminal velocities in the stellar winds of $117 \mathrm{O}$ to $\mathrm{F}$ stars from the spectra of stars in the Snow et al. 1994 Atlas of UV P Cygni Profiles. They find a discontinuity in the ratio of $\mathrm{v}_{\infty} / \mathrm{v}_{\text {esc }}$ at $21000 \mathrm{~K}$ due to a change in the nature of the lines that produce the radiation pressure. This bistability may produce a jump in the mass-loss rates of stars near $\mathrm{T}_{\text {eff }}=21000 \mathrm{~K}$ with the higher mass-loss rates at the low temperature side of the jump. Bistability may play a role in the formation of disks around $\mathrm{B}[\mathrm{e}]$ supergiants. 
Humphreys \& Davidson $(1994$, PASP 106, 1025) reviewed the luminous blue variables and discussed their brief but critical roles in massive star evolution.

Najarro et al. (1994, A\&A 285, 573) analysed the nature of the brightest galactic centre HeI emission line star. They find it very similar to the evolved blue supergiants of type Ofpe/WN9 found in the Magellanic Clouds,

Crowther et al. (1995, A\&A 302, 457) studied 3 weak lined WN3-4 stars and found that they are similar in temperature and luminosity to the strong line WN stars but have lower mass-loss rates. Smith et al. (1996, MNRAS 281, 163) present a 3-dim classification of WN stars.

Bauer \& Husfeld (1995, A\&A 300,481) describe non-LTE analyses of 7 subdwarf $O$ stars and 4 central stars of planetary nebulae. The derived abundance pattern was disparate. High gravity sdO stars have similar metal abundances with a strong signature of hydrogen burning in the CNO cycle. Luminous sdO stars show markedly different metallicities suggesting different origins.

ONC supergiants have been analysed by Smith \& Howarth (1994, A\&A 290, 868). As suggested by Walborn, OC supergiants have normal He abundances, whereas morphologically-normal $\mathrm{O}$ supergiants and ON supergiants show the products of nucleosynthesis at the surface. The Belfast group also continued work on distant B stars. In recent papers, Smartt et al. (1996, A\&AS 116, 483; A\&A 310, 113 ) studied 6 early B-type stars toward the Galactic anti-centre at distances between 10 and $18 \mathrm{kpc}$. They find that three of these stars, probably all situated within the Perseus arm show relatively normal metal abundances and that one star which is metal deficient may have formed between the Local and Perseus spiral features. Beyond a galactic radius of $12 \mathrm{kpc}$, they detect depletions in most of the stars although a significant degree of scatter is also present. A comparison between the nitrogen and oxygen differential abundances in each star shows a significant degree of scatter, whereas other reliably measured metal abundances (silicon and magnesium) appear well correlated which they think may be core CNO cycled material which has been mixed to the surfaces of some of these objects.

Be and Ae stars. Be stars continued to generate much interest. In an important paper Porter and Drew $(1995$, A\&A 296,761$)$ successfully produce an equatorial disk around a rotating $B$ star with $\mathrm{v}_{\text {rot }} / \mathrm{v}_{\text {crit }}=$ 0.25 . Line formation was considered by Hummel $(1994$, A\&A 289, 458) and Hummel \& Vrancken (1995, A\&A 302, 751). High resolution line profiles are given for 37 stars by Ballereau et al. 1995, A\&AS 111, 423). Equivalent widths from low resolution spectra are given for 42 Be stars by Mennickent et al. (1994, A\&AS 106, 427).

The structure of Be star disks is discussed on the basis of Fell emission lines in 27 Be stars by Hanuschik (1996, A\&A 308, 170) while Aerts and Molenberghs (1995, A\&A 293, 828) constrain Be star geometry using photometric and polarimetry.

Spectroscopic observations over an $18 \mathrm{yr}$ interval have been made of the binary Be star Phi Persei by Singh et al. (1994, PASJ 46, 27).

Rotation, wind and infall of Herbig Ae stars is investigated by Boehm and Catala (1995, A\&A 301, 155) and Sorelli et al. (1996, A\&A 309, 155).

Beta Pictoris. The discovery of what was claimed to be an equatorial disk and evidence of cometary debris falling into the star created great interest in $\beta$ Pic. The ultra-high resolution facility of the AAT echelle spectrograph was turned onto the CaK line by Crawford et al. (1994, MNRAS 266, L65). In their most recent paper XXII, Beust et al. (1996, A\&A 310,181) investigate the cometary infall model and suggest observations are compatible the successive infall of several bodies.

The Sun. One of the applications of the Gray line-ratio technique discussed above is the determination of the temperature variation of the sun during the solar cycle (Gray \& Livingston 1997, ApJ 474, January 10 th issue; two papers). Using the C I 5380A line, along with the adjacent lines of Fe I and Ti II, the sun is found to be 1.5 degrees hotter during the peak of the magnetic phase compared to the minimum phase. This may cause a significant re-evaluation of the hitherto accepted explanation of plage variations being the cause of the irradiance (power) variations. A slow secular trend amounting to about +0.014 degrees per year also seems to be present. This could be quite important for terrestrial climate changes if it continues over a few decades.

Non-Gaussian line profiles of emission lines from the solar chromosphere have become a current topic of some concern (e.g., Wood et al. 1996, ApJ 458, 761; Robinson et al. 1996, ApJ 469, 872; Linsky Wood $1994, \mathrm{ApJ} 430,342$ ). One of the ideas here is that the unexplained broad wings might be caused by 
micro-flaring, and if so, then micro-flares might be a significant, even dominant, source of heating for chromospheres etc.

The solar abundance of Fe was contentious: (Blackwell et al. 1995, A\&A 296, 217; Holweger et al. 1995, A\&A 296, 233; Blackwell et al. 1995 A\&A 303, 575; Kostik et al. 1996, A\&A 305, 325). But work by Milford, O'Mara \& Ross (1994, A\&A 292, 276) and Anstee, O'Mara \& Ross (1996, MNRAS in press) using collision damping constants for the strong lines from the theory of Anstee \& O'Mara (1995, MNRAS 276, 859) seems to have been able to resolve the controversy. They derive an abundance for iron of $7.51 \pm 0.01$ in detailed agreement with the meteoritic abundance of Anders \& Grevesse (1989, Geochim.Cosmochim.Acta 53, 197). Disagreement between recent analyses of the abundance based on the equivalent width of medium-strong lines can be traced to both disagreement in the basic observational and atomic data and the different microturbulent velocities employed. A sample calculation for a single line shows very clearly that a good fit to an equivalent width does not necessarily imply a good fit to the line profile and highlights the need to base analyses using lines of intermediate strength on line profiles. There was clear evidence of the effects of rapid downflows and upflows in the observed profiles which can only be accounted for by a more detailed model of the non-thermal motions in the photosphere. The sample calculation shows how it may be possible to bring abundance analyses based on lines of all strengths into agreement. The theory of Anstee \& O'Mara which accounts for the bifurcation in the solar curve-of-growth for iron (Carter effect), is a tremendous breakthrough for abundance analyses using strong lines. ¿From tables in their 1995 paper the broadening for any S-P and P-S transition for neutral elements can be calculated. They are currently completing construction of similar tables for P-D and D-P transitions. Although the same formulation does not hold for transitions for ionised elements such as CaII, they believe that minor modifications will enable the collisional broadening to be calculated for CaII H\&K and the so-called IR triplet.

Cayrel et al. (1996, A\&A 312, 549) have also used new broadening parameters to computed the profiles of the CaI triplet lines in the Sun. The broadening was taken from Spielfiedel et al. (1991, J.Phys.B. 24, 4711 ) with corrections. They obtain excellent agreement with the observations unlike the case when the classical Van der Waal's broadening was used. They confirm the proposal of Edvardsson (1988, A\&A 190, 148 ) to use these lines to derive the surface gravity of dwarfs or subgiants.

Kiselman (1994, A\&AS 104, 23) has examined high-spatial solar spectral lines in order to study the effect of granulation on the lines. The variation of line equivalent width with continuum intensity was generally linear and looks similar for different kinds of lines. No effects were found that could signal significant problems for abundance analysis using homogeneous model atmospheres.

Baumueller \& Gehren (1996, A\&A 307, 961) have investigated the formation of AlI lines in the solar spectrum. They find that the effects of non-LTE are small.

In a short note, Stenflo \& Keller $(1996$, Nature 382,588$)$ report on the spectropolarimetric spectrum of the sun, "the second solar spectrum".

Colina et al. (1996, AJ 112, 307) discuss the 0.12 to 2.5 micron absolute flux distribution of the sun and its comparison with solar analog stars, while Taylor (1994, PASP 106, 444) discussed the B-V colors of the sun. He finds that solar analogs have bluer B-V colors due to lower line blanketing below $\mathrm{H} \beta$.

Galactic Bulge. Much effort has gone into measuring the abundance and the velocities of $\mathrm{K}$ stars toward and in the Galactic Bulge. Highlights are papers by McWilliams \& Rich (1994, ApJS 91, 749): $12 \mathrm{~K}$ giants in Baade's Window; Castro et al. (1995, A\&AS 111, 17): CASPEC spectrum of 2 stars in Baade's Window; Castro et al. (1996, AJ 111, 2439): Keck HIRES observations of BW IV-167; Sadler et al (1996, AJ 112, 171): analyses of $\mathrm{K}$ giants in Baade's Window. Unpublished analyses of echelle spectra of selected strong-line stars have been carried out by Peterson, Bessell, Pfitzner and Sadler. The results from different groups are now in general agreement. There are stars in Baade's Window with abundances ranging from less than -1 to +0.4 . The most metal-rich stars have an Fe abundance similar to that of $\mu$ Leo. $\mathrm{Mg} / \mathrm{Fe}$ and $\mathrm{Ti} / \mathrm{Fe}$ are high for those stars with $\mathrm{Fe}$ abundances lower than $0.2 \mathrm{dex}$. $\mathrm{CN}$ is also very strong. $\mathrm{Ca} / \mathrm{Fe}$ and $\mathrm{Si} / \mathrm{Fe}$ follow the normal line. Some metal-rich stars have normal $\mathrm{Mg} / \mathrm{Fe}$. Do these stars belong to a different population? The mean $\mathrm{Fe}$ abundance of stars in the Bulge is $-0.25 \mathrm{dex}$. Genuine bulge $\mathrm{K}$ stars are fainter than $16 \mathrm{mag}$ in $\mathrm{V}$.

The Magellanic Clouds. Results of the first survey of the far-UV (90 - $120 \mathrm{~nm}$ ) spectra of O stars in the Magellanic Clouds made during the Astro-2 mission are presented by Walborn et al. (1995, ApJL 454, L27. Five objects in each cloud were obtained, including four O3 stars and two O4If supergiants 
with remarkably different CNO strengths. All the SMC objects are in NGC 346 and show strong effects of the low metal deficiency. One of the stars shows strong interstellar $\mathrm{H}_{2}$ features.

Interstellar extinction is a very important consideration in the Clouds. Caplan et al. (1995, A\&A 307, 403) used $\mathrm{H} \alpha$ fluxes obtained with a Fabry-perot spectrophotometer to re-calibrate the SMC photographic maps of Kennicutt \& Hodge (1988, ApJ 306, 130). These were then used in conjunction with radio-continuum maps at $843 \mathrm{MHz}$ to study the dust associated with HII regions. For most regions the derived reddenings and extinctions are compatible with the standard Galactic extinction law and uniform interstellar extinction. Oestreicher et al. $(1995$, A\&AS 112, 495) derived foreground reddenings maps at 10 ' resolution from 1409 foreground LMC stars. The foreground reddenings range from 0.00 to $0.15 \mathrm{mag}$ with a mean of $0.06 \pm 0.02 \mathrm{mag}$. Astro-2 UV polarimetry and spectrophotometry was obtained for reddened stars in the LMC by Clayton et al. (1996, ApJ 460,313). The UV interstellar polarisation shows a wavelength dependence similar to that seen in the Galaxy. The measured column density of $\mathrm{H}_{2}$ with respect to $\mathrm{HI}$ are low compared to the Galaxy and track the lower dust abundances suggesting that it may be regulated by the amount of dust present.

Spectacular HST images of Planetaries in the Clouds (Dopita et al. 1996, ApJ 460, 320) were followed by exciting HST FOS spectra (Vassiliadis et al. 1996, ApJS 105, 375). Some of their quantitative results unfortunately are affected by uncertainties in reddening corrections and uncertainties in comparisons between ground-based and FOS PRISM fluxes. Nevertheless, the $\mathrm{C} / \mathrm{O}$ ratio is anticorrelated with previously published N/O values suggesting that hot-bottom burning process operated during the AGB phase.

Leisy \& Dennefield $(1996$, A\&AS 116, 95) also present optical and UV spectroscopic observations of planetary nebulae and used them to determine carbon abundances. They discuss the CNO cycle in the precursor stars and show that $\mathrm{CN}$ or $\mathrm{ON}$ cycles are more effective with lower metallicities and are always complete for type I nebulae. From the study of $\mathrm{C}$ abundances they deduce that the 3rd dredge-up is occurring in all $\mathrm{PNe}$ and its efficiency is higher at lower metallicities hence explaining the higher number of carbon stars in metal-deficient galaxies.

Stellar abundance determinations in the Clouds are building up a more consistent picture than was evident a few years ago. Metallicities and abundances based on medium resolution spectra of $\mathrm{K}$ giants (see techniques) have been derived by Meliani et al. (1994, A\&A 290,753; A\&A 300, 349; A\&A 300, 349). They derive $[\mathrm{Fe} / \mathrm{H}]$ abundances of about -0.7 for the SMC cluster NGC 330 and the sample of SMC field stars and -0.9 for some red giants in NGC 1818 in the LMC.

In a series of excellent papers, Hill et al. (1994, A\&A 293, 347). Hill (1996, A\&A in press) and Hill, Barbuy \& Spite (1997, A\&A in press) analysed high resolution spectra of F supergiants and K supergiants in the Magellanic Clouds. The $9 \mathrm{~F}$ supergiants which were distributed across the LMC, gave surprisingly uniform deficiencies in $[\mathrm{Fe} / \mathrm{H}]$ of $-\mathbf{0 . 2 7}$. For all stars, $[\mathrm{C} / \mathrm{Fe}]<0$ but still exceed the value observed in LMC HII regions by 0.2 dex. the $[\mathrm{O} / \mathrm{Fe}]$ ratio is similar to the value in the Galactic comparison supergiant Canopus. $\mathrm{Si}, \mathrm{S}, \mathrm{Ca}$ and $\mathrm{Ti}$ are enhanced while $\mathrm{Mg}$ is slightly underabundant (compared to iron). Heavy s-and $\mathrm{r}$ process elements are overabundant by about 0.3 dex on the average, except for Eu, whose overabundance is somewhat smaller.

In the second paper, Hill derived the composition of six $\mathrm{K}$ supergiants in the SMC together with Arcturus. The SMC stars were metal deficient, with $[\mathrm{Fe} / \mathrm{H}]$ ranging from -0.6 to -0.9 dex. The $\alpha$-elements were not enhanced (unlike metal deficient stars in the Galaxy) and $\mathrm{Ni}$ was underabundant with respect to $\mathrm{Fe}$ by $0.3 \mathrm{dex}$. The $\mathrm{s}$ and $\mathrm{r}$ process elements heavier than $\mathrm{Ba}$ were enhanced by about $0.4 \mathrm{dex}$.

Finally, the CNO and $\mathrm{Li}$ abundances were derived for the six SMC K supergiants. The average $[\mathrm{O} / \mathrm{Fe}]$ ratio was -0.18 similar to that found in young objects in the $\mathrm{LMC}$ and Galaxy. The $\mathrm{C}, \mathrm{N}$ and ${ }^{12} \mathrm{C} /{ }^{13} \mathrm{C}$ ratio suggest that some mixing of CNO-cycle materials may have taken place. Li was detected in all six stars, reaching $\epsilon(\mathrm{Li})=0.6$ dex in two stars.

Nucleo-synthesis on the AGB remains one of the main interests in Cloud studies of $M$ and $C$ stars. Smith et al. (1995, ApJ 441, 735) have surveyed 112 red giants in the Magellanic Clouds for the Li 670.7 $\mathrm{nm}$ line. The Li line was detected in 35 of these stars - all AGB stars - with the majority (26) being luminous $\mathrm{S}$ stars within a narrow magnitude range $\mathrm{M}_{b o l}=-6.52 \pm 0.38$. The presence of $\mathrm{Li}$ in these highly evolved AGB stars is ascribed to hot-bottom burning.

During the past twelve years Zickgraf et al. $(1996$, A\&A 309, 505) have followed the spectroscopic and photometric behaviour of the B $[\mathrm{e}]$ star R4 in the SMC. Using high resolution spectra they found from radial velocity measurements that $\mathrm{R} 4$ is a spectroscopic binary with an early $\mathrm{B}$ component showing absorption features of SiIII, SiIV, HeI, OII and NII, and an early A-type component with absorption 
lines mainly of TiII and CrII. The emission lines of H, FeII and [FeII] lines are associated with the B star which is therefore called the $\mathrm{B}[\mathrm{e}]$ component. They derive temperatures and gravities and luminosities for the two components and from comparison with evolutionary tracks estimate a mass for the A component of $10 \mathrm{M}_{\odot}$ close to the spectroscopic mass. The ZAMS mass of the $\mathrm{B}[\mathrm{e}]$ component is about $20 \mathrm{M}_{\odot}$, that is twice the current mass. Therefore this component has obviously lost a large fraction of its mass during previous evolutionary phases. With these observations they could for the first time determine directly the mass of a $\mathrm{B}[\mathrm{e}]$ giant. The low present mass and the evolutionary timescales of both binary components show that the $\mathrm{B}[\mathrm{e}]$ giant is in an advanced evolutionary stage, most likely in the post-red supergiant phase.

Lithium, Beryllium, Boron and Deuterium. The light elements $\mathrm{Li}, \mathrm{Be}, \mathrm{B}$ and deuterium are key elements predicted to have been formed in the earliest stages of the Universe and thus offering evidence for the early densities. Unfortunately, these elements can be destroyed at quite low temperatures in stars, while $\mathrm{Li}, \mathrm{Be}$ and $\mathrm{B}$ can also be created by spallation processes in the interstellar medium or in solar-like flares on the surface of stars. Over the last 3 years a lot of effort has gone into measuring the Li abundance in a range of GK stars of different $\mathrm{Fe}$ abundance and into understanding the variation of $\mathrm{Li}$ abundance with temperature, age and other parameters. The main purpose to understand the Spite plateau in halo stars and whether this abundance reflects the primordial cosmological abundance.

Amongst the many papers, Deliyannis et al. (1994 ApJL 425, L21) found abundances in Groombridge 1830 and HD 134439 to be consistent with depletion models. They also studied a tidally coupled binary in M67 and found Li to be two time more abundant than than expected, presumably because of the rotational inhibition. $\mathrm{Li}$ abundances have also been measured in the globular cluster M92 by Deliyannis et al. (1995, ApJ 452, L13) with the Keck/HIRES. Some evidence for differences in Li abundance of three otherwise apparently identical M92 subgiants were found. This suggests that different stellar surface $\mathrm{Li}$ depletion histories in these stars from a higher initial $\mathrm{Li}$ abundance to be a more likely explanation. Pasquini \& Molaro (1996, A\&A 307, 761) have measured Li in 6 stars in the old globular cluster NGC 6397. They derive the same $\mathrm{Li}$ abundance as the field halo stars and they found no spread in the $\mathrm{Li}$ abundance of the turnoff stars. Spite et al. $(1996$, A\&A 307, 172) have reanalysed three samples of halo stars to derive the $\mathrm{Li}$ abundance. They find the scatter in all but one star fully consistent with the temperature and equivalent width errors leaving virtually no intrinsic scatter.

Lambert $(1995$, A\&A 301, 478) suggests that galactic cosmic rays decelerated in the envelopes of halo stars could make ${ }^{6} \mathrm{Li}$ and other light nucleides at observable levels. This could produce the observable amounts of ${ }^{6} \mathrm{Li}$ in extremely metal-poor stars near the main-sequence turnoff. Delyanis \& Malaney (1995, ApJ 453,810 ) also claim that flare activity could also produce the small amounts of ${ }^{6} \mathrm{Li}$. They suggest that the ratio of ${ }^{7} \mathrm{Li} /{ }^{6} \mathrm{Li}$ should be constant if a protostellar origin is the source, but the ratio should increase as a function of $\mathrm{T}_{\text {eff }}$ along the giant branch if flare production is important. Carlsson et al. (1994, A\&A 288, 860) claim the non-LTE effects for Li are significant and should be taken into account. A dip in the HK emission, similar to the one found by Boesgaard for lithium, has been discovered by Bohm-Vitense (1995 A\&A 297, L25).

Another element whose primordial abundance is sought for comparison with cosmological predictions is boron. Using the HST GHRES Edvardsson et al. (1994, A\&A 290, 176) measured the $249.67 \mathrm{~nm}$ line of $\mathrm{BI}$. They derived a B/Be ratio of about 17 in good agreement with what is expected from spallation by cosmic rays and conclude that a spallation origin for Be and B in the Early Galaxy is the most probable. The depletion of boron has also been used to test the efficiencies of mixing in main sequence $\mathrm{A}$ and $\mathrm{B}$ stars by Venn et al. (1996, A\&A 307, 849) and Fliegner et al. (1996, A\&A 308, 13). The qualitative agreement between the observed $\mathrm{N} / \mathrm{B}$ ratio and the model results provides the strongest evidence for rotational mixing acting in main sequence $B$ stars.

The correlation between light-element abundances with metallicity in metal-deficient stars has led Yoshii et al. (1995, ApJ 447, 184) to propose that the observation of a constant light-element abundance at low metallicity may not be a signature of primordial origin but rather a measure of the average accretion rate from the metal-enriched gas in the Galactic plane.

The abundances of the light elements in the interstellar medium is also of great interest. Lemoine et al. (1995, A\&A 298, 879) measured interstellar Li isotopes in the spectrum of $\zeta$ Oph. The boron isotope ratio in the interstellar medium in front of $\delta$ Sco was measured by Federman et al. (1996, Nature, 381, 764) from HST HRS spectra. ${ }^{11} \mathrm{~B} /{ }^{10} \mathrm{~B}$ was 3.4 , comparable to the value 4 in the solar system but higher than the value 2.5 expected from spallation. Of great interest (Schramm \& Turner 1996, Nature 381, 193) 
were measurements of the deuterium abundance in high-redshift hydrogen clouds seen in the spectra of a QSO spectrum taken with the Keck HIRES. More such observations are planned. The two clouds studied by Tytler et al. (1996, Nature 381, 207) have deuterium abundances relative to hydrogen of $(2.3 \pm 0.3)$ and $(2.5 \pm 0.3) \times 10^{-5}$ implying a baryon density of $\left(4.4 \pm 0.6 \times 10^{-31} \mathrm{~g} \mathrm{~cm}^{-3}\right.$. The other firm detection (Songalia et al. 1994, Nature 368, 599; Carswell et al. 1994, MNRAS 268, L1) has a much higher abundance and indicates a lower baryon density of $(1.3 \pm 0.3) \times 10^{-31} \mathrm{~g} \mathrm{~cm}^{-3}$. High abundance measurements might be due to a hydrogen interloper (different velocity) and not to deuterium. A high primeval deuterium abundance should produce a high ${ }^{3} \mathrm{He}$ abundance after processing in stars but Gloeckler \& Geiss (1996, Nature 381,210 ) find a low abundance of local ${ }^{3} \mathrm{He}$ which appears to refute a high deuterium abundance. We look forward to further observations from Keck HIRES on ${ }^{7} \mathrm{Li},{ }^{2} \mathrm{H}$ and ${ }^{3} \mathrm{He}$ that will help determine the primeval abundances of these elements unambiguously and thus tie down the baryon density.

Brown dwarfs and $M$ dwarfs. The first unequivocal discovery of a brown dwarfs was made when methane bands were discovered in the IR spectrum of the companion of GL229 by Oppenheimer et al (1995, Science 270, 1478). Excellent fits to the observed spectrum have been obtained by Tsuji et al (1996, AA 308, L29) and Allard et al (1996, ApJ 465, L123) with a gas-rich model of about 1000K.

The lithium test for brown dwarf candidates has been applied by Martin et al (1994, AA 436, 262) to 14 possible stars. All objects showed strong Li depletion as did the Keck telescope spectrum of the 23rd mag star PC0025+04447 analysed by Mould, Cohen, Oke, Reid (1996, preprint).

Jones et al. (1996, MNRAS 280, 77) have compared the $1.16-1.22$ micron spectra of $M$ dwarfs with synthetic spectra but note that quality of atomic and molecular data still severely hampers the interpretation. The chromospheres of $\mathrm{M}$ dwarfs was modelled by Houdebine et al (1995, A\&A 294, 773) who get good fits to the observed strengths of hydrogen lines.

High precision radial velocities - extra-solar planets. Numerical investigations into spectral line variations caused by non-radial pulsations has recently been published by Hatzes (1996 PASP 108, 839). This phenomenon may explain some of the recent claims of detections of extra-solar system planets. Small amplitude radial-velocity variations, resulting from line profile variations, have been detected in a number of giant stars (e.g., Walker et al. 1989, ApJ Lett 343, L21; Hatzes \& Cochran 1996 ApJ 468, 391; 1993 ApJ 413, 339). Similar studies were completed by Kennelly \& Walker (1996 PASP 108, 327).

The announcement by Mayor \& Queloz $(1995$, Nature 378, 355) of the detection of an object half the mass of Jupiter orbiting $51 \mathrm{Peg}$, has fuelled public interest in the search for other planets. Walker (1995, Nature 378, 332) summarizes work from other groups.

Halo stars - globular cluster and field stars. There have been an extraordinarily large number of papers on abundances in the extremely metal-poor population of the Galaxy over the last few years. Many of these have been stimulated by the commissioning of new high resolution echelle spectrographs and sensitive CCD systems and search programs for extremely metal-poor stars. The aim of many of the programs is to map the abundances of the heavy elements in order to facilitate comparison with $r$ - and s-process theories in the early galaxy. The abundances of the $\alpha$-elements are also of great interest as they throw light on variations in the mass function with time.

Three of the more interesting papers are Gratton \& Sneden (1994, A\&A 287, 927) - accurate heavyelement abundances in halo stars; Sneden et al (1996, ApJ 467, 819) - r-process, including Th, in rprocess-rich halo star); and Magain 1995, A\&A 297, 686 - Ba in HD 140283: s-process? The paper by Gratton \& Sneden examines 19 stars with $-2.8<[\mathrm{Fe} / \mathrm{H}]<0$ and derives the abundances of the neutron-rich elements. Some of their conclusions are that elements such as $\mathrm{Ba}$ and La thought to be mainly s-process are overdeficient compared to pure r-process elements such as Eu; there does not appear to be a plateau in abundance ratios such as $[\mathrm{Ba} / \mathrm{Eu}]$ at very low values of $[\mathrm{Fe} / \mathrm{H}]$; and the abundance pattern of neutroncapture elements in metal-poor stars show clear differences with respect to scaled solar-system r-process nucleosynthesis predictions. Sneden et al analysed an $[\mathrm{Fe} / \mathrm{H}]=-3.1$ star and determined abundances for 20 neutron capture elements. They find that the solar r-process curve when scaled provides an excellent fit to all elements in the range $56 \leq Z \leq 76$. The abundance of the cosmochronometer element thorium indicates that the star's age is at least $15.2 \pm 3.7 \mathrm{Gyr}$ - much in excess of $4.5 \mathrm{Gyrs}$. Magain enters the r/sprocess controversy looking at the barium isotopes $\mathrm{Ba}$ in HD140283 the archetype extreme metal-deficient subgiant. He finds that the barium isotopic ratio is in agreement with a pure s-process production and not r-process as suggested by Truran (1981, A\&A 97, 391). 
Some other papers of interest are Briley et al. (1995, Nature 383,604) - Na bimodality accompanies CN bimododality in 47 Tuc; Kilian-Montenbruck et al. (1994, A \& A, 291, 757) - no abundance gradient in B-stars); King (1994, ApJ 436, 331) - [O/Fe] in extreme halo stars; Nissen et al. (1994, A\&A 285, $440)-[\mathrm{O} / \mathrm{Fe}]$ in less extreme halo stars; Peterson et al. (1995, ApJ 453, 214) - rotation and OI in blue HB stars; Primas et al. (1994, A\&A 290, 885) - [m/Fe]; Kipper \& Joergensen (1994, A \& A 290, 148) - CH star; Kipper et al. (1996, A\&A 306, 489) - dwarf halo carbon stars: not all binaries; Lambert et al. (1995, ApJ 450, 3020) - s-process in AGB stars; McWilliam et al. ( 1995, AJ 109, 2757) - halo star abundances; Mishenina et al. (1995, A\&AS, 109, 471) - s-process abundances; Norris \& Da Costa (1995, ApJ 447, 680) - heavy element abundances in Omega Cen; Smith et al. (1995, AJ 110, 2827) - heavy element abundances in Omega Cen; Woolf et al. (1995, ApJ 453,660) - Eu in disk stars.

\section{Report of the Working Group on Spectroscopic Archives}

Edited from a letter by R.E.M. Griffin.

The Working Group (Spectroscopic Data) seeks to develop methodology to use very simple methods of cataloguing, recording and saving that can be adopted by all relevant observing sites. If new methodologies can be developed to make that cost-free and trouble-free for observatories for whom this is a fairly new area and where there is no local expertise and not much enthusiasm either, so much the better. One area that could be of interest to IT experts is the automatic reduction of echelle observations.

The Working Group has done a little in that I have organized a couple of meetings or sessions within conferences, but the WG has not, as far as I am aware, made much progress towards its basic aims. Recently I sent a circular asking members, among other things, to report on what they had achieved since the IAU GA, and only two replied - carefully avoiding to report anything. However, a few things have been accomplished in the name of the WG: (a) I persuaded NATO to renew our travel grant, supplying a further $\$ 4 \mathrm{~K}$. (b) One of the sessions at the Vienna Model Atmospheres meeting last summer was dedicated to 'data' in its various forms, and I lectured about archiving in general and followed it up with an open forum in which everyone came to the conclusion that it made no sense to make one's software available uncensored on the Web. I saw this as particularly germane to current discussions within CCP7 (a UK group that collects, edits and carefully disseminates software for modelling stellar spectra of various types) as to whether it should Europeanize its operations. A version of my report to the Vienna meeting is being printed in the CCP7 Newsletter. (c) The main function of the WG last year was to organise a discussion at the European Astronomical Society meeting in Catania in September. Although there were not enough of the actual WG members present to hold a business meeting, the discussion itself was rather well attended and developed into an active debate.

Another thing that $I$ have done is to write a review for Egret \& Albrecht's revision of Databases \& On-line Data in Astronomy. Since the field is rapidly evolving a new edition was foreseen on a timescale of five years, and one has recently appeared. However it does no more than update, and very patchily, some of the contributions to the first edition, while those that are sailing steadily get no mention except that the reader is referred back to edition one. This is disappointing as the subject desperately needs more than that. There is an abominable confusion over terminology, and whether or not a data (service) contains raw data, and how the most important thing for observers is to be able to scan an on-line bibliography. That sort of discussion comes from people who spend their time creating archives, not as observers or analysts using the actual data or contributing to them. Consequently I have been thinking that I would like to be able to spend time preparing a Monograph on this subject from the standpoint of the active astronomer; it will discuss rationale and present definitions, and will also describe a pilot case in which high-quality pipeline processing of echelle spectra is carried out in real time at the telescope (such a project is getting under way at the RGO).

In connection with such matters, there are two items for which I would like funding support. One is assistance to enable me to gather a minimum of US $\$ 10 \mathrm{~K}$ to begin setting up the World Plate Store in Haute-Provence, and the other is financing the time taken to prepare the Monograph on Data and Information. As described above, the latter is needed as a source of reference of (a) definitions and thelike, (2) standards for spectroscopic archives, (3) standards route(s) for achievement and (4) models or examples. CUP has agreed in principle to receive both the book and its costs, so all it wants is a couple of months' support for myself to write it and to travel in order to consult widely. I would welcome expressions of interest and support from the members of Commission 29 and the IAU. 